Review

\title{
Hepatocellular Carcinoma and Diffusion-Weighted MRI: Detection and Evaluation of Treatment Response
}

\author{
Jill S Gluskin ${ }^{1}$, Fabrizio Chegai ${ }^{\circledR}$, Serena Monti ${ }^{3}$, Ettore Squillaci² ${ }^{2}$ Lorenzo Mannelli $^{1}$ \\ 1. Department of Radiology, Memorial Sloan Kettering Cancer Center, 1275 York Avenue, C276, New York, NY 10065, USA. \\ 2. Department of Diagnostic and Molecular Imaging, Radiation Therapy and Interventional Radiology, University Hospital Tor Vergata, Viale Oxford 81, \\ 00133, Rome, Italy. \\ 3. IRCCS Fondazione SDN spa , Naples, Italy. \\ $\triangle$ Corresponding author: Fabrizio Chegai, M.D. Department of Diagnostic and Molecular Imaging, Radiation Therapy and Interventional Radiology, \\ University Hospital Tor Vergata, Rome, Italy. Viale Oxford 81, 00133 Rome Italy. Tel. 06-20902400 Email: fabrizio.chegai@libero.it.
}

(1) Ivyspring International Publisher. Reproduction is permitted for personal, noncommercial use, provided that the article is in whole, unmodified, and properly cited. See http://ivyspring.com/terms for terms and conditions.

Received: 2015.12.02; Accepted: 2016.01.22; Published: 2016.07.13

\begin{abstract}
Differentiating between cancerous tissue and healthy liver parenchyma could represent a challenge with the only conventional Magnetic Resonance (MR) imaging. Diffusion weighted imaging (DWI) exploits different tissue characteristics to conventional Magnetic Resonance Imaging (MRI) sequences that enhance hepatocellular carcinoma (HCC) detection, characterization, and post-treatment evaluation. Detection of HCC is improved by DWI, infact this technology increases conspicuity of lesions that might otherwise not be identified due to obscuration by adjacent vessels or due to low contrast between the lesion and background liver. It is important to remember that DWI combined with contrast-enhanced MRI has higher sensitivity than DWI alone, and that some patients are not eligible for use of contrast on CT and MRI; in these patients DWI has a prominent role. MRI has advanced beyond structural anatomic imaging to now showing pathophysiologic processes. DWI is a promising way to characterize lesions utilizing the inherent contrast within the liver and has the benefit of not requiring contrast injection. DWI improves detection and characterization of HCC. Proposed clinical uses for DWI include: assessing prognosis, predicting response, monitoring response to therapy, and distinguishing tumor recurrence from treatment effect. Ideally, DWI will help risk stratify patients and will participate in prognostic modeling.
\end{abstract}

Key words: Hepatocellular Carcinoma; Diffusion weighted imaging (DWI); Hepatic carcinogenesis.

\section{Introduction}

Differentiating between cancerous tissue and healthy liver parenchyma could represent a challenge with the only conventional Magnetic Resonance (MR) imaging. Diffusion weighted imaging (DWI) exploits different tissue characteristics to conventional Magnetic Resonance Imaging (MRI) sequences that enhance hepatocellular carcinoma detection, characterization, and post-treatment evaluation. By measuring diffusion of water molecules, the DWI technique is an indicator of tissue cellularity and shows promise as a prognostic imaging biomarker.

\section{DWI technique}

DWI is an MRI sequence that uses an unique imaging contrast mechanism: the diffusion of water's molecules in the tissues. Differences in the restriction of water diffusion permit differentiation between normal and abnormal tissue. Hepatocellular carcinoma (HCC) cells are smaller than normal hepatocytes ${ }^{1}$. This results in higher number of cells and cell membranes per voxel, thus restricting the diffusion of water molecules which results in higher signal intensity in HCC relative to liver parenchyma. Therefore, restricted diffusion is an indirect measure 
of tumor hypercellularity ${ }^{2-5}$. DWI takes advantage of the restriction of water motion in tumors, improving the signal contrast between tumors and background hepatic parenchyma. DWI is a T2 weighted sequence that quantitatively assesses the motion of water molecules in tissues in vivo. Diffusion weighting is determined by the b-value, expressed in seconds per square millimeter. When $b=0$, there is no diffusion weighting and the images correspond to T2 weighted images. When $b$-values increase, the images are more sensitive to diffusion, with a greater lesion-liver contrast. In clinical applications, two or more b-values are acquired for two main reasons: 1) to qualitatively assess signal intensity changes in tissues, and 2) to calculate the apparent diffusion coefficient (ADC) maps. The b-values clinically used, usually include one in the low range $(0-50 \mathrm{~s} / \mathrm{mm} 2)$ and one in the intermediate-to-high range $(400-800 \mathrm{~s} / \mathrm{mm} 2)$. More b-values can be added to calculate more accurate ADC maps and for more complex intra voxel incoherent motion (IVIM) models. Complex modeling of IVIM may have the potential for discriminating water molecules diffusion and tissue micro-perfusion; this is undergoing extensive research but has found neither unequivocal validation nor clinical applications yet. Areas of restricted diffusion, and thus high cellularity, will appear as hyperintense (bright) signal on DWI (Figure 1).

The quantification of water motion is reflected in the ADC, which is measured in square millimeter per second, best visualized as how much area a water molecule can cover per second. ADC is the post-processing of DWI sequences and an important diagnostic tool for DWI. ADC is calculated from two or more images with different $b$-values and displayed as an ADC map. Depending on the MR system and software, ADC maps may be obtained automatically or may require manual reformatting of the diffusion weighted image.

On DWI images a T2 shine through effect may happen, especially for low-to-medium b values, which are the ones used in clinical abdominal imaging. T2 shine through is when T2 hyperintense fluid remains hyperintense on DWI. Shine through is due to high amount of water within the lesion; when a lot of water is present, there may be enough water incidentally not moving or not moving fast enough to have a significant (qualitatively perceivable) decrease in signal intensity at low-to-medium $b$ values. This can be misleading. The shine through effect can be detected on ADC maps where areas of shine through on DWI will have high ADC values. Shine through effect can also be unveiled by increasing the $b$ value; however this is not practical in a clinical setting. Thus, DWI and ADC sequences are evaluated side by side to confirm if DWI hyperintensity truly indicates restricted diffusion (Figure 2). Restricted diffusion manifests as qualitative increased signal contrast between lesions and background liver on DWI which corresponds to low ADC values, thus not attributable to $\mathrm{T} 2$ shine through.

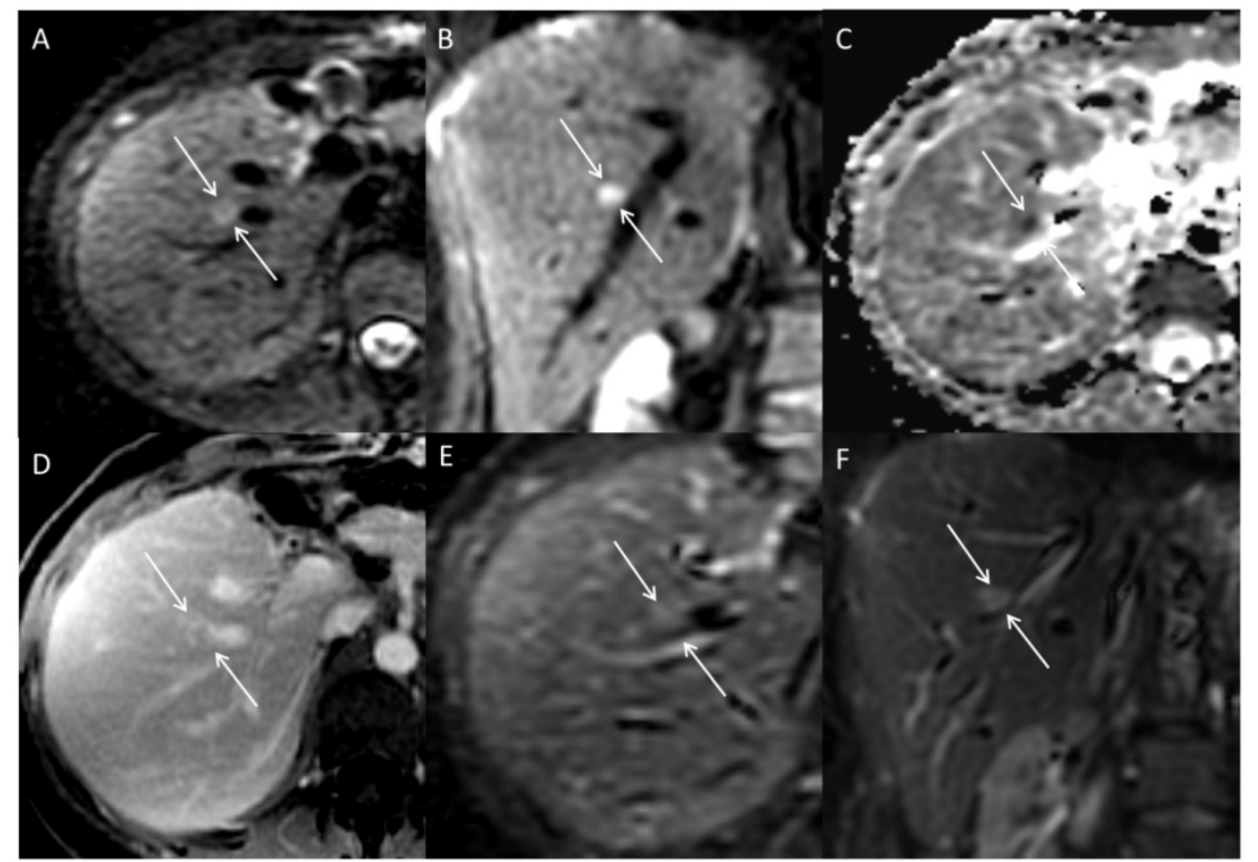

Figure 1. Multiplanar images through the liver in a patient with hepato-cholangio-carcinoma status post left hepatectomy. A lesion adjacent to a vessel is hyperintense on DWI axial (A) and coronal (B) images. The lesion is dark on ADC map (C), indicating restricted diffusion. Lesion is more apparent on DWI images than post-contrast axial (D), and T2 axial (E) and coronal (F) images; this is due to proximity of the lesion to the hepatic vein as well demonstrated in the coronal DWI (D). 


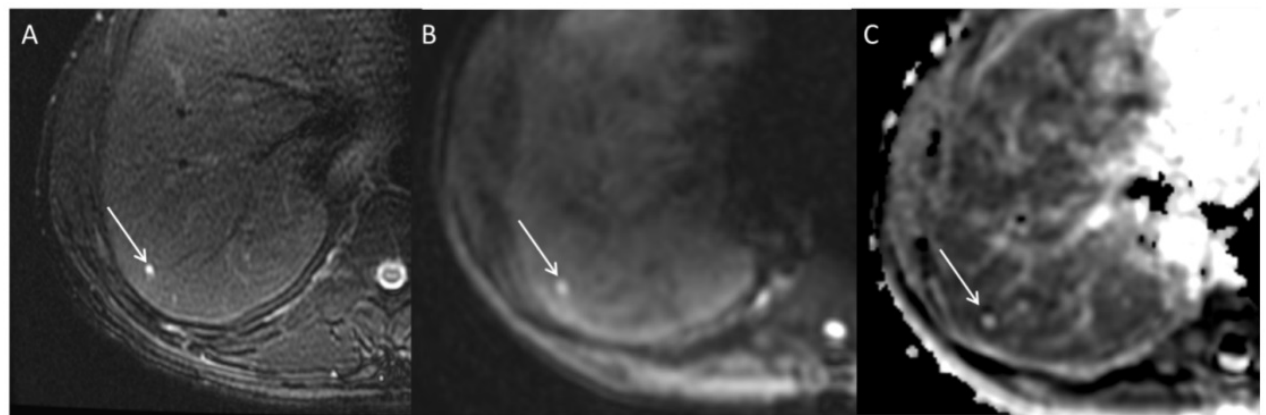

Figure 2. Axial MRI images through the liver demonstrating a small simple cyst. A subcentimeter lesion is present in hepatic segment 7; the lesion demonstrates high signal intensity on T2 weighted images (A) and b $500 \mathrm{~s} / \mathrm{mm}^{2} \mathrm{DWI}(\mathrm{B})$. However, it is bright on ADC map (C) indicating high diffusion consistent with T2 shine through phenomena. Shine through phenomena is not rare in cysts.

Using the inherent contrast within the tissue allows imaging without intravenous contrast, which is particularly relevant in patients with chronic renal failure; in fact these patients are exposed to additional risks and potential morbidity by the use of contrast media. Another benefit of DWI is its short acquisition time, which depending on the technique (breath-hold, free breathing, respiratory navigator, respiratory triggering, etc.) is usually within 2-3 minutes ${ }^{2-5}$.

There are several confounders making the quantification of lesions through DWI and ADC difficult. Average ADC values do not capture the known heterogeneity in tumors. Signal intensity is both scanner dependent, including technique and field strength, as well as patient dependent, including the inflammatory state of the liver, the amount of iron deposition, and changes in microcirculation. Therefore, no thresholds exist for DWI or ADC that are widely applied. DWI is usually acquired with eco-planar imaging (EPI) which is prone to inhomogeneity artifacts. However, DWI relies on a different contrast mechanism and spoils a different tissue characteristic to generate signal contrast between tissues. For these reasons DWI is complementary to the other MR sequences ${ }^{2-5}$.

Most HCCs are hypervascular, when compared to the background liver parenchyma. Because at lower $\mathrm{b}$ values $(0-50 \mathrm{~s} / \mathrm{mm} 2)$ decrease in signal intensity is mainly affected by micro perfusion, low $b$ values may decrease the contrast between HCCs and background liver parenchyma. Intuitively ADC measurements are affected by high vascularity of HCCs especially when low $\mathrm{b}$ values are used.

\section{Lesion detection / Diagnosis and Staging of HCC}

Detection of HCC is improved by DWI ${ }^{6,7}$. DWI increases conspicuity of lesions that might otherwise not be identified due to obscuration by adjacent vessels or due to low contrast between the lesion and background liver (Figure 1). DWI nulls the signal within vessels, which is a significant difference, compared to $\mathrm{T} 2$ and $\mathrm{T} 1$ weighted images and probably the reason why small lesions adjacent to vessels are more easily detected on DWI. DWI increases the detection rate of $\mathrm{HCC}$, particularly true for small cancers $8,9,10,11$, although some disagreement is still present on this. ${ }^{12}$ DWI assists in detection of small intrahepatic metastases by increasing the detection rate of subcentimeter tumor nodules in patients with $\mathrm{HCC}^{9}$. It is important to remember that DWI combined with contrast-enhanced MRI has higher sensitivity than DWI alone, and that some patients are not eligible for use of contrast on $\mathrm{CT}$ and MRI; in these patients DWI has a prominent role. DWI can be combined in evaluation of liver lesions; some lesions can be more evident on DWI while others can be more evident on post contrast images; however, once the lesion is uncovered in one sequence, it can be identified on the other sequences. Combining signal intensity characteristics on DWI and contrast enhanced sequences allows for a confident characterization of most of the liver lesions. An enhancing lesion associated with restricted diffusion is likely viable neoplastic tissue $10,13,14$.

DWI has imperfect sensitivity in detecting HCC (published range between 62\% and 91\%). Some HCC do not restrict diffusion or only minimally restrict diffusion, particularly lesions with well differentiated components; this is possibly due to minimal difference in the cellularity of these well differentiated HCCs and the background liver parenchyma. ${ }^{12}$ Another cause for false negative DWI is cirrhosis. Cirrhotic hepatic parenchyma causes restricted diffusion of water molecules compared to normal parenchyma. In a fibrotic liver, a HCC will have decreased lesion to liver contrast on DWI ${ }^{10,15}$. Therefore, if a lesion is suspicious for HCC on conventional MR sequences, the absence of restricted diffusion does not necessarily exclude HCC, especially if the background parenchyma is fibrotic ${ }^{16}$. Liver fibrosis and presence of regenerative and 
dysplastic nodules is a well know confounding factor for HCC detection in DWI as well as in other MRI sequences and other image modalities such as ultrasound and CT.

Regenerative and dysplastic nodules tend to accumulate iron ${ }^{17}$, resulting in decreased signal intensity on DWI which is most prominent at higher $b$ values. Decreased signal-to-noise ratio on DWI occurs because iron deposition has a strong $\mathrm{T} 2$ shortening effect and creates magnetic field inhomogeneities. Hepatocytes in HCC are usually iron-poor with iron content lower than background parenchyma, and may even be resistant to iron accumulation ${ }^{18,19}$. Iron-poor HCC therefore does not show these effects of iron deposition seen in regenerative and dysplastic nodules. If an iron-free focus develops within an iron-containing nodule, this is a sign of early neoplasia ${ }^{20}$. Another sign of neoplasia can be an iron-free focus in a liver with diffuse iron deposition.

Distinguishing bland thrombus from tumor thrombus is a promising area for DWI. Currently controversies exist concerning the utility of DWI in evaluating venous thrombus, with some authors finding DWI can differentiate tumor thrombus from bland thrombus ${ }^{21}$. Other authors have found DWI not helpful due to overlap in ADC between bland and tumor thrombus 22 . Larger prospective studies are needed to establish the value of DWI in detecting tumor thrombus.

\section{Lesion characterization}

Hypovascular nodules in patients with cirrhosis often pose a diagnostic dilemma since they can represent different etiologies, including nodular areas of fibrosis, cirrhotic nodules, low-grade or high-grade dysplastic nodules or well differentiated HCC. Cirrhotic nodules and dysplastic nodules are usually isointense on DWI. Therefore, if the differential diagnosis on conventional MR sequences is a cirrhotic nodule, a dysplastic nodule, or HCC, the presence of restricted diffusion favors a malignant etiology. In a patient with chronic liver disease, these hypovascular nodules with restricted diffusion should raise a suspicion of well differentiated HCC, or at least of a high-grade dysplastic nodule, thus helping to characterize lesions $\mathbf{7}^{7,23,14,24,25}$. Additionally, adding DWI to conventional MRI improves reader confidence $^{8}$ and accuracy ${ }^{26}$.

DWI is not specific and the restriction of water molecules can indicate a benign or malignant lesion. Small hemangiomas may restrict diffusion and be mistaken for malignancy ${ }^{27}$, although hemangiomas are often markedly, diffusely $\mathrm{T} 2$ hyperintense and usually do not pose a diagnostic dilemma. Restricted diffusion is commonly seen in malignancy and is not specific to HCC, as it is also seen in intrahepatic cholangiocarcinomas and hepatic metastases. ADC values of solid benign lesions such as FNH or adenoma can overlap with ADC values of malignant lesions ${ }^{28}$. The presence of hyperenhancement in the arterial phase and washout in the portal venous phase allow for the specific diagnosis of HCC $6,8,11,16$. In our experience the radiologist should use all sequences to try to characterize lesions detected on DWI.

\section{Role of DWI in predicting response to treatment and post-treatment lesion evaluation}

DWI has potential as a prognostic indicator in patients planning to undergo treatment for HCC, including both surgical resection and locoregional therapy. Preoperative ADC was shown as a significant risk factor for early HCC recurrence after surgical resection ${ }^{29}$. ADC of hepatocellular tumors can be predictive of response to transarterial chemoembolization (TACE), with tumors showing lower pre-treatment ADC associated with poor or incomplete response to $\mathrm{TACE}^{30}$. $\mathrm{ADC}$ is even a potential predictor of survival in patients with HCC treated with TACE; changes in ADC of tumors following TACE was shown to be an independent predictor of progression-free survival ${ }^{31}$ and overall survival $^{32}$. After intra-arterial therapy for unresectable HCC, changes in ADC and venous enhancement can stratify patients into different survival categories ${ }^{33,34}$. DWI is a promising imaging tool in predicting prognosis and response to treatments, however more investigation is required.

Evaluation of treated HCC is a common clinical challenge. Local-regional treatment of HCC results in dynamic changes and remodeling occurring at the site of treatment. A proposed clinical use for DWI includes distinguishing recurrent tumor from treatment effect. Residual or recurrent tumor manifests as low ADC values and necrotic/edematous areas show high ADC values. DWI can provide an early assessment of response to treatment, with an increase in ADC signal corresponding to the successful treatment of lesions ${ }^{35-37,38}$. Unfortunately, tumors often have mixed histology with tumor, necrosis, and granulation tissue. This produces controversial results and DWI is not always a reliable predictor of HCC recurrence compared to contrast-enhanced MRI ${ }^{39}$. Therefore, DWI should be used together with contrast-enhanced MRI and subtracted images for best results in evaluation of tumor response to treatment. 


\section{Role of DWI as a biomarker}

Hepatic carcinogenesis is a multistep process; with progression from cirrhotic nodules to dysplastic nodules to well differentiated $\mathrm{HCC}^{40}$. Hyperintensity on DWI in hypovascular nodules in patients with cirrhosis has been shown to predict progression to hypervascular $\mathrm{HCC}^{41}$.

DWI can predict the histopathologic grade of HCC. There is an inverse correlation between tumor grade and ADC value, with poorly differentiated HCC showing more restricted diffusion than well differentiated HCC $42,43,29,44-46$ although one study showed no significant association between DWI and HCC tumor grade ${ }^{47}$. DWI shows promise as an imaging biomarker, since restricted diffusion can indicate presence of progenitor cell markers ${ }^{48}$, vascular endothelial growth factor (VEGF) expression $^{49-51}$, and microvascular invasion ${ }^{52}$ (Table 1).

Table 1. Role of DWI as a biomarker.

\begin{tabular}{|c|c|c|}
\hline Tumor Grade & ADC & Potential association \\
\hline High differentiation & Good diffusion & No reported association \\
\hline Well differentiation & Moderate diffusion & No reported association \\
\hline Poor differentiation & $\begin{array}{l}\text { Restricted } \\
\text { diffusion }\end{array}$ & $\begin{array}{l}\text { presence of progenitor cell } \\
\text { markers } 1\end{array}$ \\
\hline Scarce differentiation & Scarce diffusion & $\begin{array}{l}\text { presence of progenitor cell } \\
\text { markers 1, vascular endothelial } \\
\text { growth factor (VEGF) } \\
\text { expression2-51, and } \\
\text { microvascular invasion52. }\end{array}$ \\
\hline
\end{tabular}

Ongoing research is using multiple $b$ values (even more than 10) and more complex water diffusion models in the attempt to differentiate between IVIM due to microcirculation, also referred as pseudo-diffusion, from true diffusion. Moreover current diffusion models are unable to differentiate between intra- and extra-cellular water diffusion, which may represent a substantial confounding factor in lesion characterization.

The role of DWI as a predictor of HCC pathologic grade is still under investigation. In fact the mathematical models aiming to differentiate between intravascular, extravascular, intracellular, and extravascular extracellular spaces water diffusion are still under investigation. The currently available data, on correlation between ADC values and histology should be probably interpreted as trends and not univocally explainable observations.

\section{Conclusions}

Diffusion-weighted imaging (DWI) should be considered as a functional magnetic resonance imaging (MRI) technique, useful to detect the most critical-size body malignancies: the liver is a parenchymatous organ with an important rate of malignancy development that will be advantaged from this diagnostic technique. DWI maps water diffusivity, which in HCC may be restricted as a result of changes ensuing from hepatocarcinogenesis. MRI has advanced beyond structural anatomic imaging to now showing pathophysiologic processes. DWI is a promising way to characterize lesions utilizing the inherent contrast within the liver and has the benefit of not requiring contrast injection. DWI improves detection and characterization of HCC. Proposed clinical uses for DWI include: assessing prognosis, predicting response, monitoring response to therapy, and distinguishing tumor recurrence from treatment effect. Ideally, DWI will help risk stratify patients and will participate in prognostic modeling. There is great promise that DWI will improve patient care and provide insight into tumor biology, without increasing to the healthcare general costs with respect to other commonly used imaging techniques.

In current clinical practice the main strengths of DWI are to increase the confidence in diagnosis of HCC especially when intravenous contrast administration is not possible or for small lesions adjacent to vessels.

The major limits of DWI are inconsistency in image quality, which is heavily effected by magnetic field inhomogeneity artifacts, and limited models to correlate quantitative measurements to histopathology: an important technical development could be focused on the amelioration of the image quality with a specific software able to reduce the artifacts, moreover, the radiologists would need of more reliable prognostic models to more frequently use this technique.

\section{Conflict of Interest}

All authors declare to have no conflict of interest.

\section{References}

1. Kondo F, Wada K, Kondo Y. Morphometric analysis of hepatocellular carcinoma. Virchows Archiv A, Pathological anatomy and histopathology 1988;413:425-30.

2. Herneth AM, Guccione S, Bednarski M. Apparent diffusion coefficient: a quantitative parameter for in vivo tumor characterization. European journal of radiology 2003;45:208-13.

3. Guo Y, Cai YQ, Cai ZL, et al. Differentiation of clinically benign and malignant breast lesions using diffusion-weighted imaging. Journal of magnetic resonance imaging : JMRI 2002;16:172-8.

4. Sugahara T, Korogi $\mathrm{Y}$, Kochi M, et al. Usefulness of diffusion-weighted MRI with echo-planar technique in the evaluation of cellularity in gliomas. Journal of magnetic resonance imaging : JMRI 1999;9:53-60.

5. Gauvain KM, McKinstry RC, Mukherjee P, et al. Evaluating pediatric brain tumor cellularity with diffusion-tensor imaging. AJR American journal of roentgenology 2001;177:449-54

6. Park MS, Kim S, Patel J, et al. Hepatocellular carcinoma: detection with diffusion-weighted versus contrast-enhanced magnetic resonance imaging in pretransplant patients. Hepatology (Baltimore, Md) 2012;56:140-8.

7. Xu PJ, Yan FH, Wang JH, Shan Y, Ji Y, Chen CZ. Contribution of diffusion-weighted magnetic resonance imaging in the characterization of 
hepatocellular carcinomas and dysplastic nodules in cirrhotic liver. Journal of computer assisted tomography 2010;34:506-12.

8. Le Moigne F, Durieux M, Bancel B, et al. Impact of diffusion-weighted MR imaging on the characterization of small hepatocellular carcinoma in the cirrhotic liver. Magnetic resonance imaging 2012;30:656-65.

9. Yu JS, Chung JJ, Kim JH, et al. Detection of small intrahepatic metastases of hepatocellular carcinomas using diffusion-weighted imaging: comparison with conventional dynamic MRI. Magnetic resonance imaging 2011;29:985-92.

10. Park MJ, Kim YK, Lee MW, et al. Small hepatocellular carcinomas: improved sensitivity by combining gadoxetic acid-enhanced and diffusion-weighted MR imaging patterns. Radiology 2012;264:761-70.

11. Vandecaveye V, De Keyzer F, Verslype C, et al. Diffusion-weighted MRI provides additional value to conventional dynamic contrast-enhanced MRI for detection of hepatocellular carcinoma. European radiology 2009;19:2456-66.

12. Kim YK, Kim CS, Han YM, Lee YH. Detection of liver malignancy with gadoxetic acid-enhanced MRI: is addition of diffusion-weighted MRI beneficial? Clinical radiology 2011;66:489-96.

13. Wu LM, Xu JR, Lu Q, Hua J, Chen J, Hu J. A pooled analysis of diffusion-weighted imaging in the diagnosis of hepatocellular carcinoma in chronic liver diseases. Journal of gastroenterology and hepatology 2013;28:227-34.

14. Park MJ, Kim YK, Lee MH, Lee JH. Validation of diagnostic criteria using gadoxetic acid-enhanced and diffusion-weighted MR imaging for small hepatocellular carcinoma $(<=2.0 \mathrm{~cm})$ in patients with hepatitis-induced liver cirrhosis. Acta radiologica (Stockholm, Sweden : 1987) 2013;54:127-36.

15. Taouli B, Koh DM. Diffusion-weighted MR imaging of the liver. Radiology 2010;254:47-66.

16. Khatri G, Merrick L, Miller FH. MR imaging of hepatocellular carcinoma. Magnetic resonance imaging clinics of North America 2010;18:421-50, x.

17. Zhang J, Krinsky GA. Iron-containing nodules of cirrhosis. NMR in biomedicine 2004;17:459-64.

18. Terada T, Kadoya M, Nakanuma $Y$, Matsui $O$. Iron-accumulating adenomatous hyperplastic nodule with malignant foci in the cirrhotic liver. Histopathologic, quantitative iron, and magnetic resonance imaging in vitro studies. Cancer 1990;65:1994-2000.

19. Gurusamy K. Trace element concentration in primary liver cancers--a systematic review. Biological trace element research 2007;118:191-206.

20. Pathologic diagnosis of early hepatocellular carcinoma: a report of the international consensus group for hepatocellular neoplasia. Hepatology (Baltimore, Md) 2009;49:658-64.

21. Catalano OA, Choy G, Zhu A, Hahn PF, Sahani DV. Differentiation of malignant thrombus from bland thrombus of the portal vein in patients with hepatocellular carcinoma: application of diffusion-weighted MR imaging. Radiology 2010;254:154-62.

22. Sandrasegaran K, Tahir B, Nutakki K, et al. Usefulness of conventional MRI sequences and diffusion-weighted imaging in differentiating malignant from benign portal vein thrombus in cirrhotic patients. AJR American journal of roentgenology 2013;201:1211-9.

23. Krinsky GA, Lee VS, Nguyen MT, et al. Siderotic nodules at MR imaging: regenerative or dysplastic? Journal of computer assisted tomography 2000;24:773-6.

24. Lee MH, Kim SH, Park MI, Park CK, Rhim H. Gadoxetic acid-enhanced hepatobiliary phase MRI and high-b-value diffusion-weighted imaging to distinguish well-differentiated hepatocellular carcinomas from benign nodules in patients with chronic liver disease. AJR American journal of roentgenology 2011;197:W868-75.

25. Inchingolo R, De Gaetano AM, Curione D, et al. Role of diffusion-weighted imaging, apparent diffusion coefficient and correlation with hepatobiliary phase findings in the differentiation of hepatocellular carcinoma from dysplastic nodules in cirrhotic liver. European radiology 2014.

26. Xia D, Jing J, Shen $\mathrm{H}, \mathrm{Wu}$ J. Value of diffusion-weighted magnetic resonance images for discrimination of focal benign and malignant hepatic lesions: a meta-analysis. Journal of magnetic resonance imaging : JMRI 2010;32:130-7.

27. Galea N, Cantisani V, Taouli B. Liver lesion detection and characterization: role of diffusion-weighted imaging. J Magn Reson Imaging 2013;37:1260-76

28. Miller FH, Hammond N, Siddiqi AJ, et al. Utility of diffusion-weighted MRI in distinguishing benign and malignant hepatic lesions. Journal of magnetic resonance imaging : JMRI 2010;32:138-47.

29. Nakanishi M, Chuma M, Hige $S$, et al. Relationship between diffusion-weighted magnetic resonance imaging and histological tumor grading of hepatocellular carcinoma. Annals of surgical oncology 2012;19:1302-9.

30. Mannelli L, Kim S, Hajdu CH, Babb JS, Taouli B. Serial diffusion-weighted MRI in patients with hepatocellular carcinoma: Prediction and assessment of response to transarterial chemoembolization. Preliminary experience. European journal of radiology 2013;82:577-82.

31. Vandecaveye V, Michielsen K, De Keyzer F, et al. Chemoembolization for hepatocellular carcinoma: 1-month response determined with apparent diffusion coefficient is an independent predictor of outcome. Radiology 2014;270:747-57.

32. Corona-Villalobos CP, Halappa VG, Bonekamp S, et al. Functional Magnetic Resonance Imaging Response of Targeted Tumor Burden and Its Impact on Survival in Patients With Hepatocellular Carcinoma. Investigative radiology 2014.
33. Bonekamp S, Li Z, Geschwind JF, et al. Unresectable hepatocellular carcinoma: MR imaging after intraarterial therapy. Part I. Identification and validation of volumetric functional response criteria. Radiology 2013;268:420-30.

34. Dong S, Ye XD, Yuan Z, Xu LC, Xiao XS. Relationship of apparent diffusion coefficient to survival for patients with unresectable primary hepatocellular carcinoma after chemoembolization. European journal of radiology 2012;81:472-7.

35. Mannelli L, Kim S, Hajdu CH, Babb JS, Clark TW, Taouli B. Assessment of tumor necrosis of hepatocellular carcinoma after chemoembolization: diffusion-weighted and contrast-enhanced MRI with histopathologic correlation of the explanted liver. AJR American journal of roentgenology 2009;193:1044-52

36. Bonekamp S, Jolepalem P, Lazo M, Gulsun MA, Kiraly AP, Kamel IR. Hepatocellular carcinoma: response to TACE assessed with semiautomated volumetric and functional analysis of diffusion-weighted and contrast-enhanced MR imaging data. Radiology 2011;260:752-61.

37. Yuan Z, Ye XD, Dong S, et al. Role of magnetic resonance diffusion-weighted imaging in evaluating response after chemoembolization of hepatocellular carcinoma. European journal of radiology 2010;75:e9-14

38. Schraml C, Schwenzer NF, Clasen S, et al. Navigator respiratory-triggered diffusion-weighted imaging in the follow-up after hepatic radiofrequency ablation-initial results. Journal of magnetic resonance imaging : JMRI 2009;29:1308-16.

39. Goshima S, Kanematsu M, Kondo H, et al. Evaluating local hepatocellular carcinoma recurrence post-transcatheter arterial chemoembolization: is diffusion-weighted MRI reliable as an indicator? Journal of magnetic resonance imaging : JMRI 2008;27:834-9.

40. Tatullo M, Marrelli M, Amantea M, Paduano F, Santacroce L, Gentile S, Scacco S. Bioimpedance Detection of Oral Lichen Planus Used as Preneoplastic Model. J Cancer. 2015 Aug 20;6(10):976-83. doi: 10.7150/jca.11936. eCollection 2015

41. Kim YK, Lee WJ, Park MJ, Kim SH, Rhim H, Choi D. Hypovascular hypointense nodules on hepatobiliary phase gadoxetic acid-enhanced MR images in patients with cirrhosis: potential of DW imaging in predicting progression to hypervascular HCC. Radiology 2012;265:104-14.

42. An C, Park MS, Jeon HM, et al. Prediction of the histopathological grade of hepatocellular carcinoma using qualitative diffusion-weighted, dynamic, and hepatobiliary phase MRI. European radiology 2012;22:1701-8.

43. Heo SH, Jeong YY, Shin SS, et al. Apparent diffusion coefficient value of diffusion-weighted imaging for hepatocellular carcinoma: correlation with the histologic differentiation and the expression of vascular endothelial growth factor. Korean journal of radiology : official journal of the Korean Radiological Society 2010;11:295-303.

44. Nishie A, Tajima T, Asayama Y, et al. Diagnostic performance of apparent diffusion coefficient for predicting histological grade of hepatocellular carcinoma. European journal of radiology 2011;80:e29-33.

45. Muhi A, Ichikawa T, Motosugi U, et al. High-b-value diffusion-weighted MR imaging of hepatocellular lesions: estimation of grade of malignancy of hepatocellular carcinoma. Journal of magnetic resonance imaging : JMRI 2009;30:1005-11

46. Saito K, Moriyasu F, Sugimoto K, et al. Histological grade of differentiation of hepatocellular carcinoma: comparison of the efficacy of diffusion-weighted MRI with T2-weighted imaging and angiography-assisted CT. Journal of medical imaging and radiation oncology 2012;56:261-9.

47. Nasu $\mathrm{K}$, Kuroki $\mathrm{Y}$, Tsukamoto $\mathrm{T}$, Nakajima $\mathrm{H}$, Mori $\mathrm{K}$, Minami $\mathrm{M}$. Diffusion-weighted imaging of surgically resected hepatocellular carcinoma: imaging characteristics and relationship among signal intensity, apparent diffusion coefficient, and histopathologic grade. AJR American journal of roentgenology 2009;193:438-44.

48. Jeong HT, Kim MJ, Kim YE, Park YN, Choi GH, Choi JS. MRI features of hepatocellular carcinoma expressing progenitor cell markers. Liver international : official journal of the International Association for the Study of the Liver 2012;32:430-40.

49. Huang Z, Meng X, Xiu J, et al. MR imaging in hepatocellular carcinoma: correlations between MRI features and molecular marker VEGF. Medical oncology (Northwood, London, England) 2014:31:313.

50. Tatullo M, Marrelli M, Cassetta M, Pacifici A, Stefanelli LV, Scacco S, Dipalma G, Pacifici L, Inchingolo F. Platelet Rich Fibrin (P.R.F.) in reconstructive surgery of atrophied maxillary bones: clinical and histological evaluations. Int Med Sci. 2012;9(10):872-80.

51. Inchingolo F, Tatullo M, Marrelli M, Inchingolo AM, Inchingolo AD, Dipalma G, Flace P, Girolamo F, Tarullo A, Laino L, Sabatini R, Abbinante A, Cagiano R. Regenerative surgery performed with platelet-rich plasma used in sinus lift elevation before dental implant surgery: an useful aid in healing and regeneration of bone tissue. Eur Rev Med Pharmacol Sci. 2012 Sep;16(9):1222-6.

52. Suh YJ, Kim MJ, Choi JY, Park MS, Kim KW. Preoperative prediction of the microvascular invasion of hepatocellular carcinoma with diffusion-weighted imaging. Liver transplantation : official publication of the American Association for the Study of Liver Diseases and the International Liver Transplantation Society 2012;18:1171-8. 